\title{
Stress-Endurable Temperature Sensor Designed for Temperature Compensation on a Pressure Sensor
}

\author{
Young-Soon Moon, In-Cheol Ryu ${ }^{1}$, Won-Ho Son², \\ Si-Hun Lee and Sie-Young $\mathrm{Choi}^{2, *}$ \\ Department of Sensor and Display Engineering, \\ Kyungpook National University, Daegu 702-701, Korea \\ 'Department of Engineering Technology Electronic Materials Engineering, \\ Kyungpook National University, Daegu 702-701, Korea \\ ${ }^{2}$ School of Electronics Engineering, Kyungpook National University, Daegu 702-701, Korea
}

(Received July 11, 2014; accepted December 10, 2014)

Key words: temperature sensor, thermistor, MEMS, silicon strain gauge, pressure sensor

In this paper, the mechanical properties and temperature characteristics of a temperature sensor are presented for effective temperature compensation of a pressure sensor with a silicon strain gauge. A p-type diffused resistor of $\langle 100\rangle$ direction on the (001) plane acts as a temperature sensor because its resistance varies with temperature. Unlike typical temperature sensors, the sensor's resistance is not affected by the stress on the diaphragm caused by its deformation under pressure. Thus, the temperature sensor is able to provide the correct resistance value corresponding to the temperature, regardless of the position of the diaphragm. Only $0.0062 \%$ of the resistance change of the temperature sensor is attributable to the mechanical stress of the diaphragm's deformation.

\section{Introduction}

Semiconductor materials have a high gauge factor and offer advantages in miniaturization. Single-crystal silicon is widely used as a sensing material for pressure sensors based on the piezoresistive effect. ${ }^{(1)}$ However, these pressure sensors require temperature compensation because the resistance of the sensors is highly sensitive to temperature changes. ${ }^{(2,3)}$ However, piezoresistive temperature sensors, typically, are also sensitive to changes in mechanical stress. The purpose of this research is to fabricate a temperature sensor whose response is independent of mechanical stress.

The pressure sensor consists of two parts, a spring element and a sensing element. The spring element is a center boss diaphragm made from SUS630 in order to withstand sudden high-pressure transients and to improve the nonlinearity of the sensor output. ${ }^{(4,5)}$ A silicon strain gauge (SSG) is used as a sensing element to detect the deformation of the

${ }^{*}$ Corresponding author: e-mail: sychoi@ee.knu.ac.kr 
diaphragm and to output an electrical signal that is proportional to the applied pressure. ${ }^{(6,7)}$ A temperature sensor, designed to be insensitive to changes in its mechanical stress, is implanted in the center of the SSG for measuring the precise temperature at the closest possible proximity to the pressure-sensing element.

\section{Physical Principles}

When a force per unit area is applied to the diaphragm of a sensor, the diaphragm is deformed and a strain appears on its surface. The stress on an attached or embedded strain gauge is caused by the strain on the diaphragm. As a result, the resistance of the piezoresistors in the strain gauge is changed. From the theory of plates and shells, the pressure causes a radial strain $\left(\varepsilon_{\mathrm{r}}\right)$ and a tangential strain $\left(\varepsilon_{\mathrm{t}}\right)$ to act on the membrane. ${ }^{(8,9)}$

In the case of plane stress, the stress on a strain gauge is calculated on the basis of Hooke's law within the elastic deformation range of the material. The components of the strain, $\varepsilon_{11}, \varepsilon_{22}$, and $\varepsilon_{33}$, denote the radial strain on the diaphragm, the tangential strain on the diaphragm, and the thickness strain on the diaphragm, respectively. Note that $\varepsilon_{33}$ will be nonzero because of its Poisson's ratio. Stress $\left(\sigma_{i j}\right)$ is expressed in terms of strains $\left(\varepsilon_{k l}\right)$ by the tensor in eq. (1):

$$
\sigma_{i j}=c_{i j k l} \varepsilon_{k l} \text {, }
$$

where $c_{i j k l}$ is the elastic stiffness tensor that corresponds to Young's modulus under the uniaxial condition.

The single-crystal silicon of the sensing element is an anisotropic material. The tensor is expressed by matrix notation as ${ }^{(10-12)}$

$$
\left[\begin{array}{c}
\sigma_{11} \\
\sigma_{22} \\
0 \\
0 \\
0 \\
\sigma_{12}
\end{array}\right]=\left[\begin{array}{cccccc}
c_{11} & c_{12} & c_{12} & 0 & 0 & 0 \\
c_{12} & c_{11} & c_{12} & 0 & 0 & 0 \\
c_{12} & c_{12} & c_{11} & 0 & 0 & 0 \\
0 & 0 & 0 & c_{44} & 0 & 0 \\
0 & 0 & 0 & 0 & c_{44} & 0 \\
0 & 0 & 0 & 0 & 0 & c_{44}
\end{array}\right]\left[\begin{array}{c}
\varepsilon_{11} \\
\varepsilon_{22} \\
\varepsilon_{33} \\
0 \\
0 \\
\varepsilon_{12}
\end{array}\right] .
$$

When stress is applied to single-crystal silicon, the resistance change is dominated by the change in resistivity. ${ }^{(13-15)}$ The resistance change of an arbitrary primed axis is expressed by

$$
\frac{\Delta R}{R}=\frac{\Delta \rho}{\rho_{0}}=\sigma_{1} \pi_{1}+\sigma_{\mathrm{t}} \pi_{\mathrm{t}}
$$

where $\rho_{0}$ is the resistivity and $\sigma_{1}, \sigma_{\mathrm{t}}, \pi_{1}$, and $\pi_{\mathrm{t}}$ are the longitudinal stress, transverse stress, longitudinal piezoresistance coefficient, and transverse piezoresistance coefficient, respectively. 
The crystallographic point group of silicon in the cubic system is $m 3 \mathrm{~m} \cdot .^{(13,16)}$ In the cubic system with the symmetry of silicon, three unique piezoresistive coefficients, $\pi_{11}$, $\pi_{12}$, and $\pi_{44}$, remain as independent matrix components, as shown by

$$
\frac{1}{\rho}\left[\begin{array}{c}
\Delta \rho_{1} \\
\Delta \rho_{2} \\
\Delta \rho_{3} \\
0 \\
0 \\
\Delta \rho_{6}
\end{array}\right]=\left[\begin{array}{cccccc}
\pi_{11} & \pi_{12} & \pi_{12} & 0 & 0 & 0 \\
\pi_{12} & \pi_{11} & \pi_{12} & 0 & 0 & 0 \\
\pi_{12} & \pi_{12} & \pi_{11} & 0 & 0 & 0 \\
0 & 0 & 0 & \pi_{44} & 0 & 0 \\
0 & 0 & 0 & 0 & \pi_{44} & 0 \\
0 & 0 & 0 & 0 & 0 & \pi_{44}
\end{array}\right] \cdot\left[\begin{array}{c}
\sigma_{1} \\
\sigma_{2} \\
0 \\
0 \\
0 \\
\sigma_{6}
\end{array}\right] .
$$

If the Cartesian coordinate system is aligned to the $<110>$ equivalent direction of the piezoresistor by transformation from the crystal axes at $45^{\circ}$, then the three piezoresistor orientations exhibit the following relationships between resistance changes and stresses in the SSG. ${ }^{(16,17)}$

$$
\begin{gathered}
\frac{\Delta R_{1}}{R_{1}}=\left(\frac{\pi_{11}+\pi_{12}+\pi_{44}}{2}\right) \sigma_{11}^{\prime}+\left(\frac{\pi_{11}+\pi_{12}-\pi_{44}}{2}\right) \sigma_{22}^{\prime}+\alpha^{\prime} \Delta T \\
\frac{\Delta R_{\mathrm{t}}}{R_{\mathrm{t}}}=\left(\frac{\pi_{11}+\pi_{12}-\pi_{44}}{2}\right) \sigma_{11}^{\prime}+\left(\frac{\pi_{11}+\pi_{12}+\pi_{44}}{2}\right) \sigma_{22}^{\prime}+\alpha^{\prime} \Delta T \\
\frac{\Delta R_{1}}{R_{1}}=\left(\frac{\pi_{11}+\pi_{12}}{2}\right)\left(\sigma_{11}^{\prime}+\sigma_{22}^{\prime}\right)+\left(\pi_{11}-\pi_{12}\right) \sigma_{12}^{\prime}+\alpha^{\prime} \Delta T
\end{gathered}
$$

In the case of a p-type piezoresistor, the longitudinal piezoresistive coefficient $\left(\pi_{1}\right)$, parallel to the direction of electric current in the $<110>$ direction, is $1 / 2\left(\pi_{11}+\pi_{12}+\pi_{44}\right)$, and the transverse piezoresistive coefficient $\left(\pi_{t}\right)$, perpendicular to the electric current direction, is $1 / 2\left(\pi_{11}+\pi_{12}-\pi_{44}\right){ }^{\text {(1) }}$ The coefficient of piezoresistivity exhibits a maximum value in the $\langle 110\rangle$ equivalent direction and a minimum value in the $\langle 100\rangle$ equivalent direction. To achieve maximum sensitivity of the pressure sensor, the piezoresistors are aligned in the $\langle 110\rangle$ direction, and to detect only the temperature variation, the temperature sensor is placed in the $\langle 100\rangle$ direction. Figure 1 shows the crystal orientation of the diffused resistors and the direction of current and stress. The resistance of a diffused resistor is also changed by its temperature. ${ }^{(18,19)}$ The sensitivity of the temperature dependence of resistance is represented by

$$
\alpha=\frac{R-R_{0}}{R_{0} \cdot\left(T-T_{0}\right)}=\frac{\Delta \rho}{\rho_{0} \Delta T},
$$

where $\alpha$ is the temperature coefficient of resistance (TCR). 


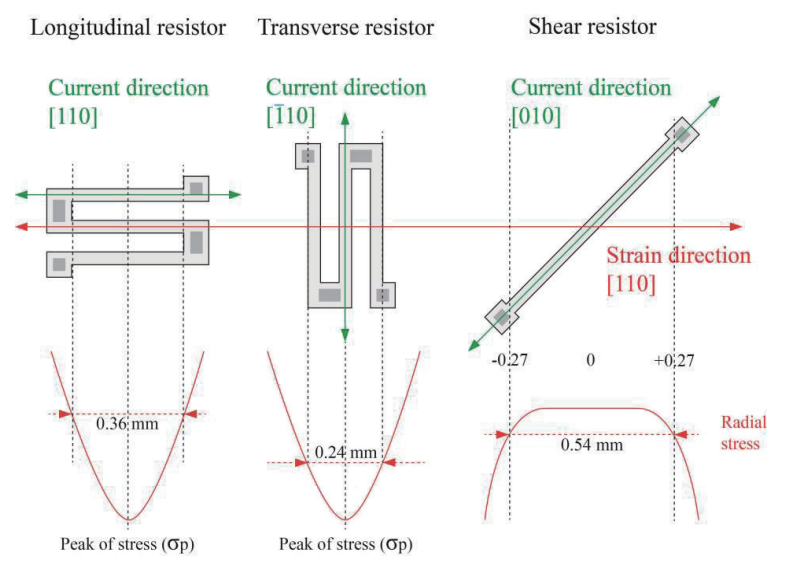

Fig. 1. (Color online) Crystal orientation of the diffused resistor.

\section{Experiment}

\subsection{Silicon strain gauge}

The SSG was designed as a linear type that is easy to align in an assembly process, as shown in Fig. 2. Two pairs of piezoresistors in the SSG are located at the rim of the diaphragm. $R_{1}$ and $R_{3}$ are longitudinal resistors whose resistance is reduced by the compressive strain. The resistance changes of the transverse resistors $R_{2}$ and $R_{4}$ are the opposite of those of $R_{1}$ and $R_{3}$. The temperature resistor $\left(R_{\text {Temp }}\right)$ is a shear resistor.

The SSG was fabricated using the following micro-electromechanical systems (MEMS) process. The original oxide layer of the wafers was removed by initial cleaning, and the silicon oxide was grown to $5000 \AA$ in a wet furnace. The piezoresistors and the $\mathrm{R}_{\text {Temp }}$ were patterned in the $\langle 110\rangle$ and $<100>$ directions, respectively. The p-type diffused resistors were fabricated by ion implantation of boron at a dose of $2.5 \times 10^{15}$ ions $/ \mathrm{cm}^{2}$, an energy of $70 \mathrm{keV}$, and a drive-in at $1000{ }^{\circ} \mathrm{C}$ for $60 \mathrm{~min}$ in wet atmosphere. The surface impurity concentration is $1.27 \times 10^{19}$ ions $/ \mathrm{cm}^{3}$. When this step was completed, the contact windows were opened. For use as an electrode, $\mathrm{NiCr} / \mathrm{Au}$ was deposited to $1500 \AA$ using a thermal evaporator. The SSG was processed by chemicalmechanical planarization (CMP) to a thickness of $80 \mu \mathrm{m}$ in order to effectively detect the deformation of the diaphragm. Finally, the wafer was cut to the size of the SSG by a dicing saw. The length and width of each SSG are 14 and $1.2 \mathrm{~mm}$, respectively.

The piezoresistive response and temperature dependence of the diffused resistors are determined by the doping concentration. As the doping concentration increases, the piezoresistive effect and the temperature coefficient of the resistance decrease. ${ }^{(20)}$

\subsection{Diaphragm}

The spring element of the pressure sensor was designed with a bossed diaphragm to reduce the nonlinearity in the sensor output. SUS630 bossed diaphragm of $10 \mathrm{~mm}$ diameter were produced by computer numerical control (CNC) and include a center boss of $2 \mathrm{~mm}$ diameter and $0.4 \mathrm{~mm}$ thickness, as shown in Fig. 3. 

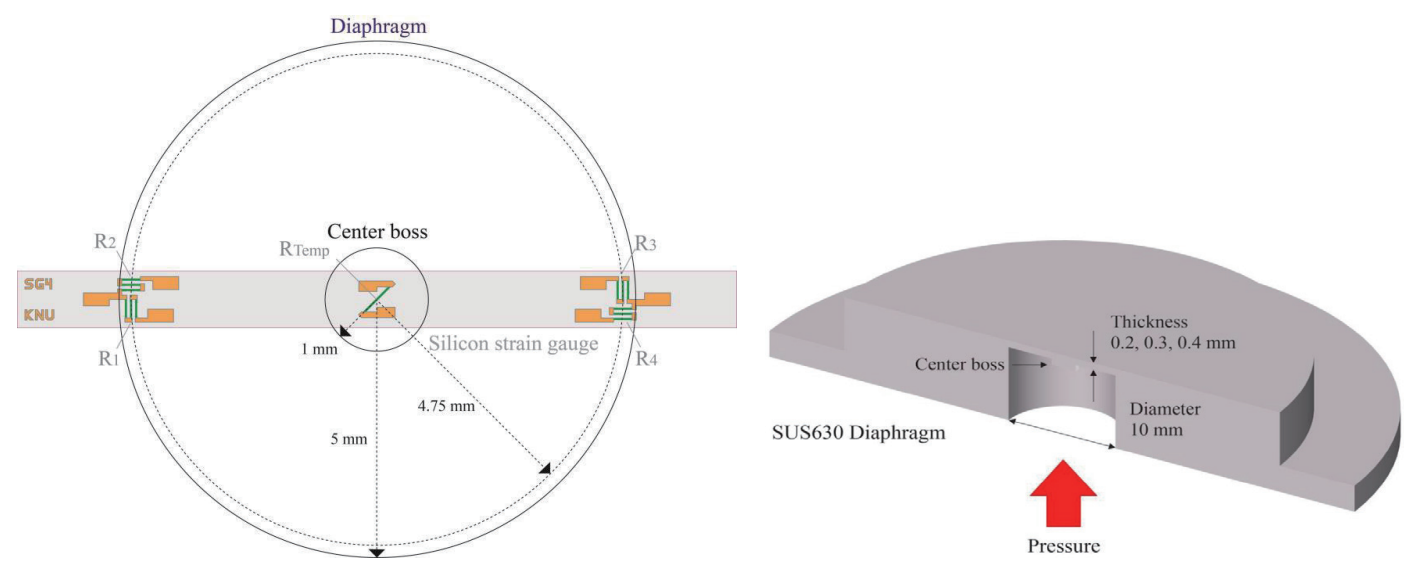

Fig. 2 (left). (Color online) Schematic diagram of the silicon strain gauge.

Fig. 3 (right). (Color online) Schematic diagram of the SUS630 bossed diaphragm.

The fabricated SSG was attached to the center of the diaphragm using M-bond 600 . The attachment process is critical for the sensitivity of the pressure sensor, because the SSG should be deformed by the same strain as the diaphragm under a given pressure. The diffused resistor and printed circuit board (PCB) of the electrode are connected by wire bonding. A silicone rubber coating protects the sensing element and wires.

In the specimen for the determination of the TCR of the diffused resistors, the SSG was aligned in the same crystal orientation as the silicon substrate, as shown in Fig. 4(a). The pressure sensor, with an SSG installed on an SUS630 bossed diaphragm, is shown in Fig. 4(b). The measuring equipment used in the experiment is shown in Fig. 5. The temperature difference is measured inside an oven according to the position of the measurement sample; thus, the temperature is adjusted on the basis of the resistance of the platinum resistance thermometer (PT100) for the reference temperature sensor.

\section{Results and Discussion}

Figure 6 shows the change in resistance of the diffused resistors in the SSG at $20{ }^{\circ} \mathrm{C}$ as the pressure applied to the pressure sensor $(200 \mu \mathrm{m}$ diaphragm thickness $)$ is increased from 0 to 10 bar. The average change in resistance of the longitudinal resistors $R_{2}$ and $R_{4}$ are $-7.9957 \%$ and $11.6360 \%$ for the transverse resistors $R_{1}$ and $R_{3}$. The resistance change of the $\mathrm{R}_{\text {Temp }}$ is only $0.0062 \%$. Note that the $\mathrm{R}_{\text {Temp }}$ has almost no response to the stress that is applied on the diaphragm by pressure.

To examine the temperature characteristics of the diffused resistors of the strain gauge according to the thermal expansion coefficients of different types of substrate, the temperature was changed by $\Delta T=90{ }^{\circ} \mathrm{C}$. On the silicon substrate, which has the same thermal expansion coefficient as the SSG, the resistance of the piezoresistors is changed by $6.8525 \%$, and that of $\mathrm{R}_{\text {Temp }}$ is changed by $7.4104 \%$, as shown in Fig. 7(a). The temperature characteristic of the SSG in the pressure sensor with a diaphragm thickness 


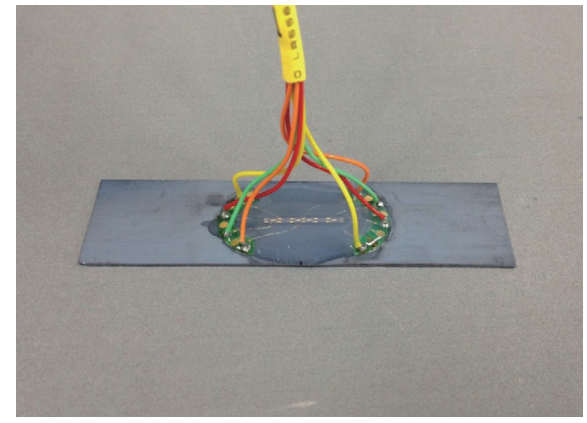

(a)

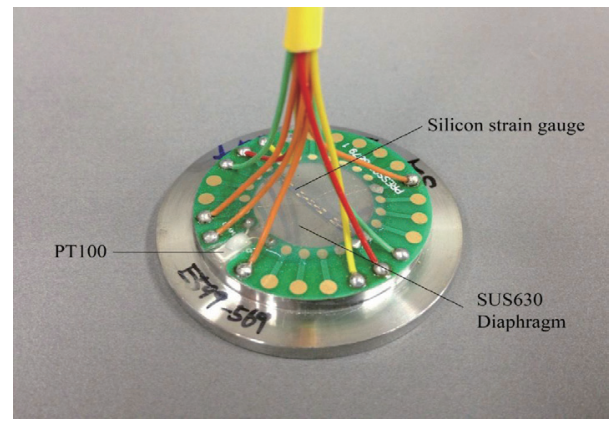

(b)

Fig. 4. (Color online) Temperature characteristics of a piezoresistor according to the substrate: (a) silicon substrate and (b) SUS630 bossed diaphragm.
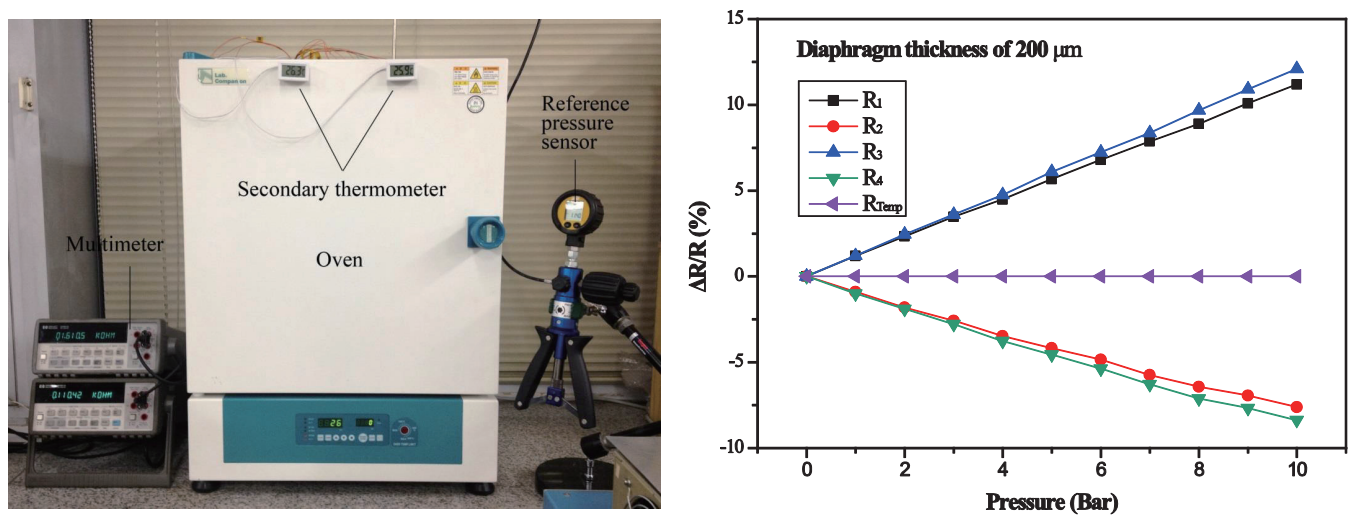

Fig. 5 (left). (Color online) Measuring equipment for the experiment.

Fig. 6 (right). (Color online) Change in resistance of the diffused resistors as a function of pressure.

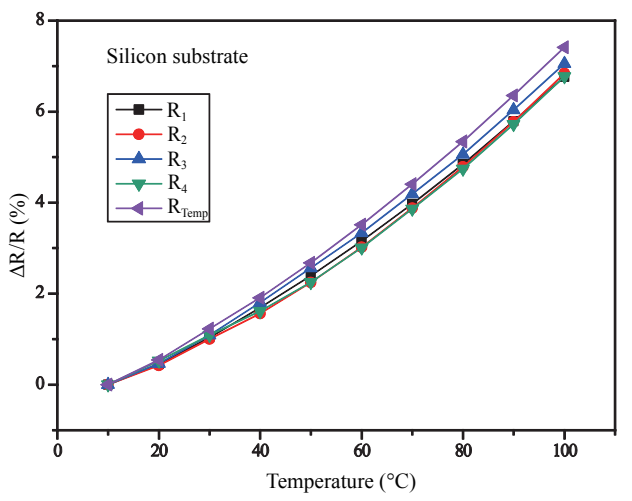

(a)

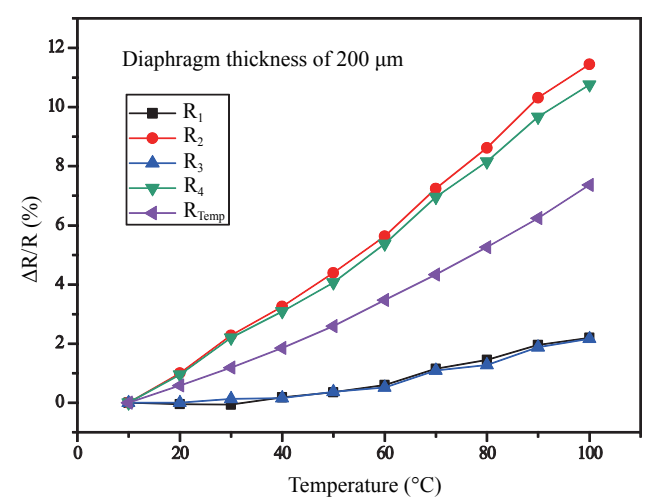

(b)

Fig. 7. (Color online) Resistance of the diffused resistors as a function of temperature $\left(T_{0}=10^{\circ} \mathrm{C}\right)$ : (a) Silicon strain gauge on a silicon substrate and (b) silicon strain gauge on the SUS360 diaphragm. 
of $200 \mu \mathrm{m}$ is investigated under the no pressure condition; changes in resistance of the diffused resistors are shown in Fig. 7(b). The average changes in resistance of the longitudinal resistors $\mathrm{R}_{2}$ and $\mathrm{R}_{4}$ are $11.0972 \%$ and $2.1799 \%$ for the transverse resistors $R_{1}$ and $R_{3}$; for $R_{\text {Temp }}$, it is $7.3670 \%$. The change in resistance of the longitudinal resistors is observed to be relatively large as compared with the changes in the resistance of other resistors because the stress acts on the diaphragm as tensile stress from the difference in the coefficient of thermal expansion between the sensing element and the spring element. However, the rate of resistance variation of $\mathrm{R}_{\text {Temp }}$ is nearly the same for both types of substrate.

The amount of resistance change was measured for each diffused resistor according to the temperature when the pressure of the sensor is changed by 10 bar, as shown in Fig. 8 . The amount of resistance change for the longitudinal resistors is reduced by $52.4160 \%$ and that of the transverse resistors is reduced by $58.7951 \%$. The average resistance change for $\mathrm{R}_{\text {Temp }}$ is $0.0158 \%$ and the standard deviation is $6.1 \times 10^{-3}$.

The thickness of the diaphragm affects the stress because of the difference in thermal expansion. Figure 9(a) shows the temperature characteristics of the longitudinal resistors. The average rate of change in longitudinal resistance varies depending on the thickness of the diaphragm. In the case of $\mathrm{R}_{\text {Temp }}$, there is no difference according to the thickness of the diaphragm, as shown in Fig. 9(b). It also appears that the resistance of $\mathrm{R}_{\text {Temp }}$ is not changed by the difference in thermal expansion coefficient between silicon and SUS630.

The TCR is calculated for a specific temperature range, as shown in Fig. 10. The average TCR of $\mathrm{R}_{\text {Temp }}$ is $609.17 \mathrm{ppm} /{ }^{\circ} \mathrm{C}$ at $25{ }^{\circ} \mathrm{C}$, which corresponds to room temperature, and the TCR increases with temperature. The sensitivity of the temperature dependence of the diffused resistor depends on the surface doping concentration.

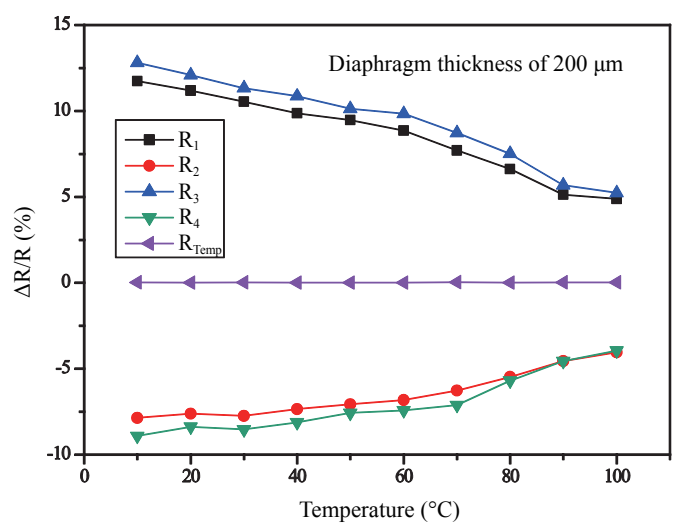

Fig. 8. (Color online) Amount of change in resistance of the diffused resistors as a function of temperature ( $\triangle P=10$ bar $)$. 


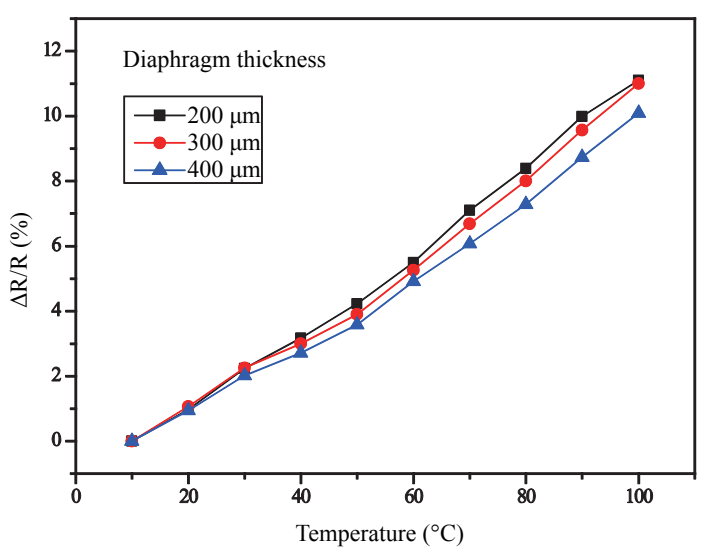

(a)

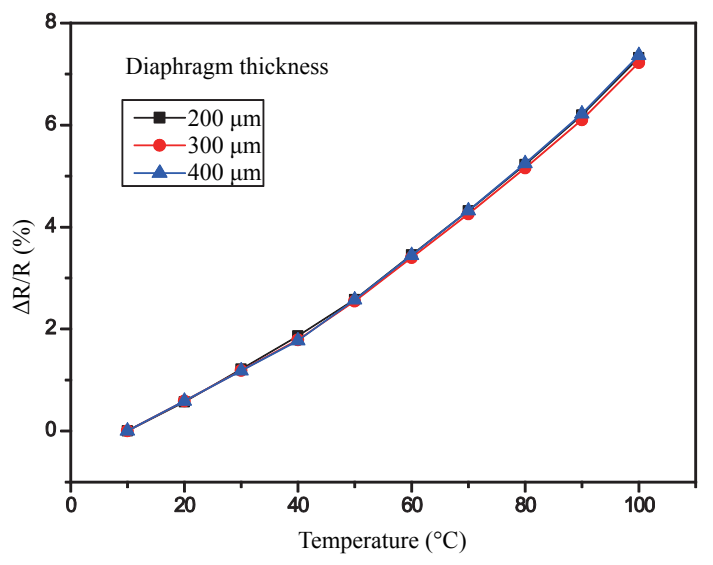

(b)

Fig. 9. (Color online) Temperature characteristics of a resistor according to the thickness of the diaphragm: (a) Changes in resistance of the longitudinal resistors and (b) changes in resistance of the $\mathrm{R}_{\text {Temp }}$.

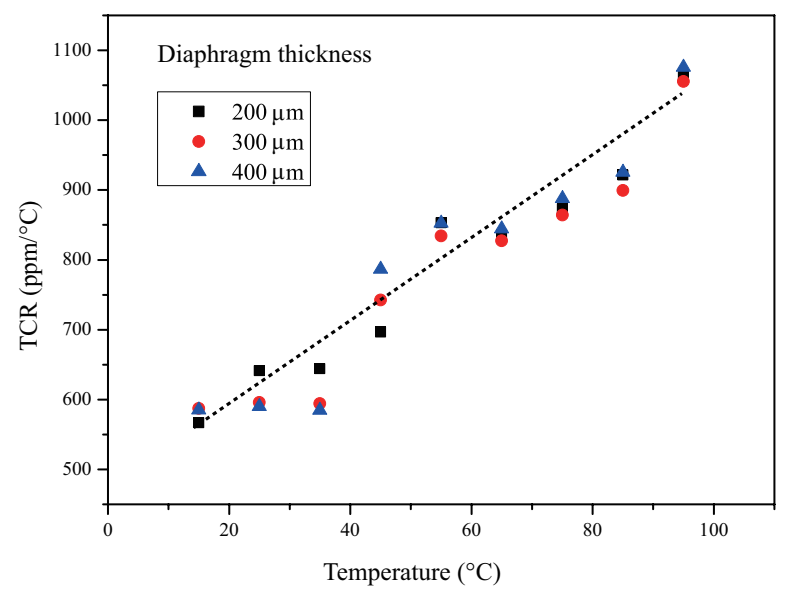

Fig. 10. (Color online) TCRs of the $\mathrm{R}_{\text {Temp }}$ at each temperature $\left(T_{0}=T_{\mathrm{m}}, T=T_{\mathrm{m}}+10^{\circ} \mathrm{C}\right)$.

\section{Conclusions}

The temperature sensor that was developed for temperature compensation of a pressure sensor has essentially no response to the mechanical stress of the sensor. The temperature sensor generates almost the same resistance under mechanical and thermal stresses. The average TCR of the thermistor is $609.17 \mathrm{ppm} /{ }^{\circ} \mathrm{C}$ at $25{ }^{\circ} \mathrm{C}$. Regardless of the change in stress, the temperature sensor is able to provide an accurate measurement of temperature at the closest possible proximity to the pressure-sensing element. 


\section{Acknowledgements}

This study was supported by the BK21 Plus funded by the Ministry of Education, Korea (21A20131600011).

\section{References}

1 C. S. Smith: Phys. Rev. 94 (1954) 42.

2 W. K. Schomburg, Z. Rummler, P. Shao, K Wulff and L. Xie: J. Micromech. Microeng. 14 (2004) 1101.

3 Y. Kanda: IEEE Trans. Electron Devices ED-29 (1982) 64.

4 A. Yayasukaya, M. Shimazoe and Y. Matsuoke: IEEE Trans. Electron Devices 36 (1989) 1295.

5 Y. Kanda and A. Yasukawa: Sens. Actuators, A 62 (1997) 539.

6 N. M. White and J. E. Brignell: Sens. Actuators, A 25-27 (1991) 313.

7 S. G. Lee, Y. S. Moon, W. H. Son, S. Y. Kwon and S. Y. Choi: ICEIC 2013, S. J. Ko (IEEE, Bali, 2013) p. 664.

8 S. P. Timoshenko and S. Woinowsky-Krieger: Theory of Plates and Shells (McGraw-Hill, Singapore, 1959) 2nd ed., Chap. 3.

9 O. N. Tufte and E. L. Stelzer: J. Appl. Phys. 34 (1963) 313.

10 M. A. Hopcroft, W. D. Nix and T. W. Kenny: J. Microelectromech. Syst. 19 (2010) 229.

11 J. Turley and G. Sines: J. Phys. D: Appl. Phys. 4 (1971) 264.

12 J. J. Wortman and R. A. Evans: J. Appl. Phys. 36 (1965) 153.

13 Y. Kanda: Sens. Actuators, A 28 (1991) 83.

14 H. P. Phan, D. V. Dao, P. Tanner, L. Wang, N. T. Nguyen, Y. Zhu and S. Dimitrijev: Appl. Phys. Lett. 104 (2014) 111905_1.

15 S. M. Sze: Semiconductor Sensors (Wiley, New York, 1994) 1st ed., Chap. 4.

16 J. C. Suhling and R. C. Jaeger: IEEE Sens. J. 1 (2001) 14.

17 C. H. Cho, R. C. Jaeger and J. C. Suhling: IEEE Sens. J. 8 (2008) 1455.

18 A. Boukabache and P. Pons: Electron. Lett. 38 (2002) 342.

19 W. M. Bullis, F. H. Brewer, C. D. Kolstad and L. J. Swartzendruber: Solid-State Electron. 11 (1968) 639.

20 O. N. Tufte and E. L. Stelzer: Phys. Rev. 133 (1964) A1705. 